\title{
Matthias Schöning Was es bedeutet, gegen Journalisten zu hetzen
}

\author{
Ein Blick in die Literaturgeschichte von Gustav Freytag bis \\ Josef Haslinger
}

https://doi.org/10.1515/iasl-2018-0003

\begin{abstract}
The problematization of journalistic work is a recurring theme in literature. This paper traces the reservations journalists see themselves confronted with according to literary representations, how criticism is solidified, and how the suspicion that journalists are making up facts is becoming more universal. This will be done by looking at a historical line of texts, ranging from Die Journalisten by Gustav Freytag to Josef Haslinger's Opernball. There are two main criteria underlying the choice of texts: first, the figures of journalists play a central role in each of the texts; second, these texts make visible the stages of a presumed development process from the middle of the $19^{\text {th }}$ century through to today in the most succinct way possible.
\end{abstract}

Angesichts der zunehmenden Hetze gegen Journalisten, journalistische Arbeit oder einfach ,die Medien' ist es an der Zeit, nach einer wichtigen Voraussetzung $\mathrm{zu}$ fragen: Was disponiert Journalisten eigentlich zum Feindbild? Oder umgekehrt: Wieso erscheint es politischen Akteuren Erfolg versprechend, ausgerechnet den Journalismus zu attackieren? Sind sie selbst das originäre Ziel oder gilt der Hass etwas anderem, für das sie - gleichgültig, ob das den Beteiligten bewusst ist oder nicht - stellvertretend gebrandmarkt werden?

Ein Blick in die Literaturgeschichte, hier primär die deutschsprachige, hilft weiter. Literarische Texte, das heißt genauer Dramen und Romane oder Erzählungen mit fiktionalem Anspruch und fiktiven Handlungen, liefern zwar zu keiner Zeit ein präzises Spiegelbild realer Verhältnisse. Aber sie zeichnen mittels Zuspitzung oder auch Verzerrung scharfe Porträts typischer Situationen und exemplarischer Akteure. Die Literatur spiegelt nicht die Wirklichkeit, aber sie

PD Dr. Matthias Schöning, Universität Konstanz, Fachbereich Literaturwissenschaften, Universitätsstraße 10, 78464 Konstanz, E-Mail: Matthias.Schoening@uni-konstanz.de 
archiviert die Bilder, die man sich - historisch und sozial differenziert - von ihr macht.

Dabei können literarische Texte Stereotype ebenso kultivieren und weitertragen wie der Kritik unterziehen. Will man die Literatur nicht mystifizieren, muss man wohl mit beiden Möglichkeiten rechnen. Nützlich sein kann im Rückblick aber beides, die helle wie die dunkle Seite des literarischen Leistungsspektrums. Im besten Fall liefert sie anschauliches Material für Gegenwartsfragen und wird nachträglich nützlich, wenn für das historiografische Bohren dicker Bretter keine Zeit bleibt. Zugegeben, das ist weder eine besonders elaborierte noch feinsinnige und keinesfalls umfassende Sicht auf Literatur, aber immerhin eine recht robuste Annahme. Wer von der Literatur erwartet, dass sie Anschauungsmaterial auch für nicht-poetische Fragen liefert, wird selten enttäuscht.

Nicht verschwiegen werden soll auch, dass der vorliegende Text die ausformulierte Form des eher tabellarischen Schlussresümees eines Hauptseminars des Verfassers an der Universität Konstanz darstellt. Es stand unter der sich gelegentlich artikulierenden Skepsis gegenüber historischen Gegenständen. Der Frage, warum solche ,alten Sachen' zu lesen seien, wo es doch drängende Probleme gebe, sollte mit zwei einander flankierenden Antworten begegnet werden. Einerseits ist $\mathrm{zu}$ bemerken, dass das Alte oft weniger alt ist, als man meint. Andererseits ist festzustellen, dass man historische Gegenstände oft unbefangener prüft als aktuelle. Man kann an ihnen also lernen, was uns auch heute bewegt, ohne sich zum Bewegenden unmittelbar ins Verhältnis zu setzen und direkt eine Meinung haben zu müssen. Es hat daher ,Methode', dass die bearbeiteten Texte, die auch wegen ihrer Handhabbarkeit im Seminar ausgewählt wurden, hier wie dort nicht von literaturwissenschaftlicher Forschungsliteratur umstellt wurden. Schließlich war ja der Beweis anzutreten, dass schon genaue Textlektüren Erkenntnisse befördern.

Für die vorliegende Frage nach der politischen Feindbildtauglichkeit des Journalismus jedenfalls lohnt ein Blick in die ausgewählten Texte aus der Geschichte der Literatur seit Mitte des 19. Jahrhunderts. Man sieht dann auf den ersten Blick starke Kontinuitäten. Schmähungen, wie sie sich im Kampfbegriff ,Lügenpresse' artikulieren, haben eine durchaus lange Geschichte und sind eine Erblast nicht erst des Nationalsozialismus. Vielmehr begleitet der Verdacht auf Unglaubwürdigkeit und Unzuverlässigkeit den Berufsstand der Journalisten von Beginn an. Und dass der Vorbehalt gegen Parlamentarismus und Öffentlichkeit ihn ebenso lange wie ein Schatten begleitet, gemahnt daran, sich die Konstitution der Moderne in Erinnerung zu rufen, mit der alle drei koevolutionär verbunden sind. Das Wissen um eine solche longue durée hilft, um in der Hitze der Gegenwartsdebatten gelassen zu bleiben.

Zudem macht die historische Horizontbildung deutlich, in welcher Hinsicht die jüngere Zeit das Problem vertieft und bis zur heute als dringlich empfundenen 
Lage verschärft. Dabei denkt die Literaturgeschichtsschreibung natürlich eher in Jahrzehnten. Allzu aktualistische Antworten darf man nicht erwarten, doch selbst das kann helfen. Durch den mehrfach gestaffelten Vergleichshintergrund werden unterschiedliche Dimensionen eines Phänomens deutlich. Die Gegenwart erhält Kontur, ohne immer wieder nur mit den Zwanzigerjahren verglichen werden zu müssen - was stets alarmistisch klingt.

\section{Auftakt - Honoré de Balzac: Verlorene Illusionen}

Als eine Art Prolog darf zunächst Honoré de Balzacs dreiteiliger Roman Illusions perdues nicht übergangen werden. In dessen Mittelteil Un grand homme de Province à Paris aus dem Jahr 1839 findet man alle klassischen Vorwürfe, die man dem Journalismus und dem Journalisten-Milieu nur machen kann, in grellsten Farben dargestellt. Im Kontrast zu einer Gruppe redlicher Dichter, Romanciers und Künstler stellt Balzac, der eigens eine „Typenlehre der Pariser Presse“ ausgearbeitet hat, ${ }^{1}$ die Journalisten nicht nur als entlaufene Schriftsteller, sondern vor allem übelste Schufte dar. Die gerade erst erworbenen Freunde, die Lucien, die Hauptfigur, ihrer Armut zum Trotz vor dem Überlaufen zum Journalismus abhalten wollen, lassen keinen Zweifel daran, dass „Korruption“ sein Wesen sei: ,aus System feig und niederträchtig [...] damit hast du den Journalismus in zwei Worten“.2

Wie sich sogleich zeigt, sind Journalisten sich für nichts zu schade, um ihr sittenloses Leben zu finanzieren. Anstatt unabhängig zu bleiben wie die Künstler um die Gegenfigur des Daniel d'Arthez, ${ }^{3}$ fügen sie sich in eine milieuspezifische Ökonomie. Die ihrem Sumpf eigenen ,Naturalien', mit denen sie stapelweise bestochen werden, sind bei Rezensenten Freiexemplare und bei Theaterkritikern die Eintrittskarten, die in schwunghaftem Handel über Zwischenhändler weiterverkauft werden. Bei politischen Journalisten sind die Zahlungsmittel Posten und Titel. Doch man ist nicht nur bestechlich. Der Vorwurf lautet eigentlich, dass Journalisten den nacktesten persönlichen Vorteil allen anderen Kriterien vorzie-

1 Diese ist kürzlich erstmalig in deutscher Sprache erschienen, was die Aktualität noch einmal unterstreichen mag: Honoré de Balzac: Von Edelfedern, Phrasendreschern und Schmierfinken. Die schrägen Typen der Journaille. Hg. und übersetzt von Rudolf von Bitter. Zürich: Manesse 2016. Die „Typenlehre“, Balzacs ursprüngliche Monographie de la presse parisienne, stellt darin den ersten Teil dar.

2 Honoré de Balzac: Verlorene Illusionen. Übersetzt von Otto Flake. Zürich: Diogenes 1977, S. 313.

3 Vgl. Balzac: Verlorene Illusionen (Anm. 2), S. 287ff. 
hen. Wenn irgendwo unter zivilen Bedingungen die Metapher vom Menschen, der dem Menschen ein Wolf ist, seine Berechtigung hat, dann hier. Jeder versucht jeden jederzeit zu übervorteilen oder ganz zu Fall zu bringen. Allianzen haben eine kurze Laufzeit. Verlass ist auf niemanden in diesem Milieu zwischen Schauspielerinnen, die sich prostituieren, und Politikern, die von Intriganten nicht zu unterscheiden sind. Alle Ambition erschöpft sich darin, der menschlichen Triebstruktur Nahrung zu geben.

Angesichts der Vielzahl von Verfehlungen, die Balzac seiner Journalistenhorde zuschreibt, verliert man fast aus den Augen, was hier historisch signifikant sein könnte. Es scheint zur Paradoxie seiner Comédie humaine zu gehören, den genauen Blick für sozial und historisch spezifische Verhaltensformen auf der Basis einer weniger anthropologischen als zoologischen Theorie zu gewinnen. In seiner Vorrede zur Comédie humaine schreibt Balzac explizit:

Es gibt nur ein Tier. Für alle organischen Wesen hat sich der Schöpfer immer nur eines Modells bedient. Das Tier ist ein Prinzip, das seine äußere Gestalt, oder genauer: seine Spezifikationen in der Umwelt, in dem Milieu annimmt, in dem es sich zu entwickeln hat. $^{4}$

Auch Journalisten sind demnach Tiere, ohne dass damit schon eine Wertung verbunden wäre. Die Einsicht, die durch die Relation von Spezies und Milieu gewonnen wird, ist um den Preis der Enthistorisierung jedoch teuer erkauft. Dass das Milieu ein spezifisch historisches ist und einer Spezies die aus einer sozialgeschichtlichen Lage resultierenden Formen vermittelt, bleibt unausgesprochen.

Der Erkenntnisgewinn kann nichtsdestotrotz hoch sein. Zwei Merkmale von Journalisten beziehungsweise literarischer Journalisten-Figuren haben topische Qualität und begleiten die literarische Darstellung von nun an auch dort, wo ein helleres Licht auf den Berufsstand des Journalisten fällt. Erstens und eher folkloristisch sind Journalisten - in den Augen von offensichtlich keineswegs unbefangenen Schriftstellern - gescheiterte Literaten und tendieren zur Boheme-Existenz. Zweitens wechseln Journalisten typischerweise die Seite und das heißt im politischen Spektrum, das seit der Französischen Revolution vertraut ist, von links nach rechts oder umgekehrt.

Eigens rekonstruiert werden muss, dass hinter diesen vordergründigen Merkmalen eine ambivalente Funktionalität am Werk ist. Der Journalismus stellt die öffentliche Meinung her, die all jene Personen brauchen, die von Popularität

4 Honoré de Balzac: Vorrede zur ,Menschlichen Komödie‘. Übersetzt von Claudia Schmölders. In: H.B. (Hg.): Die menschliche Komödie. Supplement. Zürich: Diogenes 1998, S. 7-27, hier S. 8. 
abhängig sind. Vom Beginn seiner literarischen Darstellung an ist die Leistung des Journalismus, nämlich Öffentlichkeit, Ergebnis einer Konstruktion. Zu erkennen ist außerdem, dass diese konstruktivistische Arbeit des Journalismus konstitutiv illoyal gegenüber Personen ist. Die einzelnen Nachrichten und auch die Publikationsorgane werden zwar von Menschen gemacht, aber nicht für Menschen. Journalismus entfaltet sich erst dort, wo anonyme Kommunikation unverzichtbar ist, das heißt in Großstädten und Flächenstaaten, die nicht mehr von Fürstenhöfen oder ähnlichen Interaktionsforen aus zentral regiert werden. Seine Adresse sind unübersichtliche Gesellschaften, die zu groß sind, um sich mittels persönlicher Anschauung ein Bild von der Lage zu machen. Im Unterschied zu Angoulême und seinem adeligen Salon, dem Ausgangsort des Protagonisten, führt Balzacs Paris vor Augen, warum es eine öffentliche Meinung braucht, in der sich die politische Stimmung spiegelt.

\section{Gustav Freytag: Die Journalisten}

Wenn man von einem moralistischen in ein gesellschaftstheoretisches Register wechselt, macht es also durchaus Sinn, dass die Figuren, die dem Journalismus in der literarischen Darstellung ein Gesicht geben, oftmals mehr oder minder ehrlos sind und öfter die Seiten wechseln. Unter den gegebenen Bedingungen ist das kein persönlicher Makel, sondern ein Merkmal, das den Stand der gesellschaftsgeschichtlichen Entwicklung anzeigt. Mit wachsender Anonymität der sozialen Verhältnisse geht es immer weniger darum, dass ein Schreiber wirklich glaubt, was er behauptet. Wie sollte man das auch überprüfen, wenn man sich doch nie begegnet? Viel wichtiger ist die gesellschaftliche Funktion des Journalismus im Ganzen, die Aufgabe, den unaufhebbaren Plural an Meinungen und Interessen zur Sprache zu bringen.

Wer Journalisten persönlich angreift, hat nicht verstanden, worum es geht - eigentlich: Mit dieser Einschränkung verlagert sich das Problem auf die Seite der Beobachter, also all derjenigen, die über Journalismus sprechen, von Politikern über Literaten bis zu Literaturwissenschaftlern, Soziologen und anderen Beobachtern dritter Ordnung, die die Schraube noch einmal weiter drehen. Wie man an der Literatur seit Balzac nämlich auch sehen kann, ist es sehr schwierig, ausschließlich mit abstrakten Beschreibungen zurechtzukommen. Abstraktion geht trivialerweise mit dem Verzicht auf Konkretion einher. Wer sich auf sie einlässt, merkt schnell, dass es schwieriger wird, mit dem Finger auf ,tatsächlich Schuldige‘ zu zeigen. Das schützt zwar einerseits vor übereilten Urteilen und kann zu einem Gewinn an Gerechtigkeit führen, aber es besteht andererseits auch die Gefahr, dass wichtige Bedürfnisse wie die Be- 
nennung von Verantwortlichen, die „Zuschreibung von agency“, ${ }^{5}$ unerfüllt bleiben.

An Gustav Freytags Komödie Die Journalisten aus dem Jahr 1854 lässt sich zeigen, was es heißt, die Verhältnisse mit schwankender Genauigkeit darzustellen. Verglichen mit dem Paris Balzacs bringt Freytag eine Kleinstadtidylle auf die Bühne. Genrespezifisch erhält jeder, was er verdient. Am Ende steht nicht nur die Versöhnung aller Guten, auch wenn sie zwischenzeitlich auf Abwegen waren, sondern sogar eine Doppelhochzeit. Und die eine Braut heiratet auch noch unter Stand und schenkt ihrer wiedergewonnenen ,Kinderliebe ${ }^{6}$ die Zeitung, ${ }^{6}$ in der die Hauptfigur, ihr Bräutigam, vor Beginn der Bühnenhandlung gezeigt hat, was in ihm steckt, nämlich die Fähigkeit, Mehrheiten zu organisieren. Auf der Bühne sehen wir davon nur noch die heiße Phase, in der sich die öffentlichen Angelegenheiten mit den privaten zunächst unheilvoll verknüpfen und schließlich so aufgelöst werden, dass die konservative Fraktion unredlicher Machenschaften überführt und beim Ringen um die einflussreiche Zeitung düpiert wird.

Doch so kurios das Hochzeitsfinale unter Zeitungsmachern auch wirken mag, es taugt nicht zur Denunziation des Dargestellten. Politik und Journalismus sind wie selbstverständlich verknüpft. Sie gehören zusammen und wer sie auseinanderreißen will, der tut das nicht, weil er Korruption befürchtet, sondern die Zeit zurückdrehen will. Insofern geht es zunächst einmal um die „Berechtigung des parlamentarischen Lebens überhaupt“ “. Differenziert wird intern, unter der Voraussetzung, dass es ohne öffentliche Debatte keine Politik und letztlich auch keinen Fortschritt geben kann. Leitend ist dann im zweiten Schritt die Alternative zwischen fairen und unfairen Strategien bei der unverzichtbaren politischen Meinungsmache. Wahlkampf und Journalismus dürfen voneinander profitieren, wenn sie nicht gar - wie in jener Entwicklungsphase der Journalismusgeschichte offensichtlich - zusammengehören. Parteipolitik und parteinehmender Journalismus sind zwei parallel auftretende Symptome einer Moderne, die sich von patriarchalischen Konsensfiktionen verabschieden musste, weil anders die Interessen zunächst des Bürgertums und später der Arbeiterschaft nicht zur Geltung zu bringen sind.

5 Vgl. Albrecht Koschorke: Wahrheit und Erfindung. Grundzüge einer Allgemeinen Erzähltheorie. Frankfurt/M.: Fischer 2012, S. 79ff. (Kap. „Zuschreibung von agency“).

6 Vgl. zu diesem wiederkehrenden Motiv der Realisten Sebastian Susteck: Kinderlieben. Studien zum Wissen des 19. Jahrhunderts und zum deutschsprachigen Realismus von Stifter, Keller, Storm und anderen. Berlin/New York: De Gruyter 2010.

7 Bruce Beaton: Gustav Freytags ,Die Journalisten'. Eine politische Komödie der Revolutionszeit. In: Zeitschrift für deutsche Philologie (ZfdPh) 106 (1986), S. 516-543, hier S. 531. 
Die gleichsam funktionalistische Privilegierung des Konflikts gegenüber dem Konsens fällt jedoch schwer. Jeder meint doch, selbst auf der richtigen Seite zu stehen. Wenn man nicht gleich in einen ironischen Indifferentismus wechseln will, sind Zugeständnisse allenfalls in Verfahrensfragen sinnvoll. Und so legt auch das Drama des moderaten Reformers Gustav Freytag nahe, dass der prinzipiell befürwortete Meinungskampf mit fairen Mitteln ausgetragen werden kann und soll. Um das seinen Zuschauern und Lesern nahezubringen, schreibt Freytag bei der Konzeption der Figuren und Handlungen der Gruppe der moderaten Reformpolitiker einen Stil der persönlichen Aufrichtigkeit zu. In Verknüpfung mit den Darstellungsgrenzen der realistischen Dramaturgie zieht diese Übersetzung von Verfahrensfragen in Aufrichtigkeitsfragen jedoch nach sich, dass die Apologie moderner Verhältnisse in lediglich semi-moderner Gestalt daherkommt. Von einer längeren Kneipen- und kürzeren Mauerschau-Szene abgesehen, sieht man nur Innenseiten von Politik und Journalismus, also persönliche Konflikte und keine öffentlichen Räume. Und dabei stehen sich auch noch ein dominant redliches Lager und ein dominant unredliches gegenüber. Das Aushängeschild der unfair operierenden Konservativen, der Oberst a.D. Berg, eigentlich ein Muster an Integrität und Gemeinsinn, ist den Unredlichen nur zwischenzeitlich auf den Leim gegangen. Als er begreift, dass man seine Ambitionen als Leitartikler bloß instrumentalisiert hat, zieht er sich zurück. Die oppositionellen Lager sind am Ende wieder reinlich getrennt.

Am Oberst wird ex negativo vorgeführt, dass Persönlichkeit kein Faktor journalistischer Professionalität ist. Zum Witz der Komödie trägt nicht zuletzt bei, dass er nur mühsam versteht, dass die publizistischen Reaktionen auf seine anonym publizierten Texte nicht ihm persönlich gelten (können). Explizit darf jedoch nur eine Randfigur an die Konstitutionsbedingungen des Journalismus erinnern. Der Jude Schmock, mehr als alle anderen Journalisten vom Zeilenhonorar existenziell abhängig, will die Seiten wechseln und darf einen Satz mit langem historischen Nachhall sprechen: „Ich habe bei dem Blumenberg gelernt, in allen Richtungen zu schreiben. Ich habe geschrieben links und wieder rechts. Ich kann schreiben nach jeder Richtung. “8

Schmock will zu den Fortschrittlichen überlaufen. Und als man sich dort bedenklich gibt und das politische "Gewissen“ ins Spiel bringt," erinnert Schmock an die Regeln - und zwar Figuren wie Zuschauer gleichermaßen. Kurzgefasst lautet die Grundregel moderner Kommunikation unter den Bedingungen von Schriftlichkeit und Druck, dass schon bei journalistischer Arbeit, der Tages-

8 Gustav Freytag: Die Journalisten. Lustspiel in vier Akten. Stuttgart: Reclam 1988, S. 46.

9 Freytag: Die Journalisten (Anm. 8), S. 46. 
aktualität gegebenenfalls zum Trotz, die Situationsabstraktheit schriftlicher Kommunikation greift und die Bedeutung eines Textes sich vom Verfasser ablöst. ${ }^{10}$ Freytag beweist damit, dass er, selbst erfahrener Publizist, ${ }^{11}$ nicht so naiv ist, wie sein Stück phasenweise denken lassen könnte. Doch ähnlich wie in seinem Roman Soll und Haben, einem der wichtigsten Bücher für das Selbstverständnis des deutschen Bürgertums in der zweiten Hälfte des 19. Jahrhunderts, überlässt er es weitgehend den Juden-Figuren, ${ }^{12}$ die unliebsame Kehrseite der gefeierten Modernisierung explizit zu machen. ${ }^{13}$

Es ist viel darüber geschrieben worden, wie das zu bewerten ist. ${ }^{14}$ War Freytag Antisemit oder nicht und wenn ja, inwieweit? Doch unabhängig davon dürfte eines unstrittig sein. Indem Freytag einen Juden und Überläufer daran erinnern lässt, dass die primäre Anforderung an Journalisten nicht Authentizität ist, sondern die Fähigkeit, Öffentlichkeit herzustellen durch Plausibilisierung relevanter Meinungen, bietet er seinen Zuschauern und Lesern eine Hintertür zur Flucht aus der Modernität, der er doch das Wort redet. Die objektive Ambivalenz der Modernisierung reduziert sich auf die Ambivalenz des Juden Schmock. Wer ihm die

10 Vgl. Manfred Frank: Was ist ein literarischer Text, und was heißt es, ihn zu verstehen? In: Jutta Kolkenbrock-Netz/Gerhard Plumpe/Hans Joachim Schrimpf (Hg.): Wege der Literaturwissenschaft. Bonn: Bouvier 1985, S. 10-25, hier S. 13, vgl. auch S. 17.

11 Vgl. Bernt Ture von zur Mühlen: Gustav Freytag. Biographie. Göttingen: Wallstein 2016, S. $100 \mathrm{ff}$.

12 Wenn es nicht direkt als Credo eines Überläufers formuliert wird, tragen die anderen Journalisten-Figuren die Darstellung journalistischer Arbeit als einer konstruktiven Tätigkeit - konstruktiv hier nicht als Wertbegriff, sondern im Sinne von ,Konstruktivismus' verstanden - mit. Vgl. Freytag: Die Journalisten (Anm. 8), S. 19: „Bolz: [...]. Erfinde deine eigenen Geschichten, wozu bist $\mathrm{Du}$ Journalist?“

13 Robert Theel: Kommunikationsstörungen. Gustav Freytags Kritik an Parteipresse und Politikgeschäft in seinem Lustspiel ,Die Journalisten‘. In: Euphorion 90 (1996), S. 185-205, sieht Freytag ein eher negatives Bild zeichnen, das bezüglich des Journalismus eine insgesamt „schwindende Authentizität der durch Medien gesteuerten öffentlichen Meinung“ diagnostiziert (S. 193). Figurenkonstellation und Handlungsentwicklung wird das jedoch nur schwerlich gerecht. Seine Interpretation, die als Leitdifferenz des Textes die Trennung von Privatsphäre und Öffentlichkeit ausmachen zu können meint, legt sich durch ihre Thesenbildung von vornherein darauf fest, im Journalismus ein Agens der Korruption zu erblicken. Zwar ist zuzugestehen, dass es Freytag nicht gelingt, die Konstitution von Öffentlichkeit mit der Unmittelbarkeit dramatischer Darstellung tatsächlich vorzuführen. Die Herstellung von Öffentlichkeit durch Meinungsmacher beziehungsweise entsprechende Handlungen dominieren die Darstellung durchgängig und werden mit verschiedenen Facetten ausgeführt. Dass der Konflikt durch persönliche Nebenumstände wie Heiratsabsichten über Lagergrenzen hinweg verschärft wird, ändert daran nichts.

14 Vgl. insbesondere die Monografie Martin Gubser: Literarischer Antisemitismus. Untersuchungen zu Gustav Freytag und anderen bürgerlichen Schriftstellern des 19. Jahrhunderts. Göttingen: Wallstein 1998, und die seither erschienenen Aufsätze. 
zitierte Äußerung als charakteristisch jüdische Eigenschaft zuschreibt, darf weiter glauben, man könne in das Zeitalter periodisch wechselnder Regierungen und unabschließbarer Meinungsbildungsprozesse eintreten, ohne die Übersichtlichkeit kleinstädtischer Verhältnisse einzubüßen. Freytags Apologie von Journalismus und politischer Repräsentation hat damit einen klar benennbaren Preis, nämlich die Kultivierung des Mythos, dass sich jeder vor Ort selbst ein Bild von seiner Welt machen kann, ohne Vermittler zu benötigen. Und dazu gehört als Kehrseite der Medaille ein latenter Antisemitismus, der alle Formen der Uneigentlichkeit als generische Eigenschaft von Juden darstellt.

\section{Arthur Schnitzler: Fink und Fliederbusch}

Unter den genannten Umständen ist es ein Wagnis, dass Arthur Schnitzler noch im Ersten Weltkrieg das Problem in einer Komödie mit dem Titel Fink und Fliederbusch frontal angeht. ${ }^{15}$ Doch der Autor ist ein erfahrener Kämpfer gegen die Tradierung hohl gewordener Rituale und hat genug leidvolle Erfahrungen mit antisemitischen Stereotypen, ${ }^{16}$ um zu wissen, was er tut. Zudem lag es offensichtlich in der Luft, das Stereotyp vom links und rechts schreibenden Journalisten aufzunehmen, wie ein zeitgleich entstehendes Drama von Tristan Bernard, Les deux canards, beweist. ${ }^{17}$ Vielleicht reicht es aus, sich 1916/1917 - das heißt dem Erstaufführungs- beziehungsweise Publikationsjahr des Dramas - daran zu erinnern, wie manche der wortgewaltigen Intellektuellen noch kurz vor Beginn des Weltkriegs gesprochen haben, um die geringe Halbwertzeit vermeintlich fester Überzeugungen zu kennen.

15 Vgl. Arthur Schnitzler: Fink und Fliederbusch. In: A.S.: Komödie der Worte. Dramen 19141916. Frankfurt/M.: Fischer 1993, S. 117-226. Zur Relation von Journalismus und Judentum und zu den intertextuellen Bezügen zu Freytags Drama vgl. Sigurd Paul Scheichl: Juden, die keine Juden sind. Die Figuren in Schnitzlers Fink und Fliederbusch. In: Mark H. Gelber/Hans Otto Horch/ S.P.S. (Hg.): Von Franzos zu Canetti. Jüdische Autoren aus Österreich. Neue Studien. Tübingen: Niemeyer 1996, S. 225-238.

16 Dazu gehört vor allem die Auseinandersetzung um seine Novelle Lieutenant Gustl. Vgl. Ursula Renner: Dokumentation eines Skandals. Arthur Schnitzlers ,Lieutenant Gustl‘. In: HofmannsthalJahrbuch zur europäischen Moderne 15 (2007), S. 33-216.

17 Vgl. Wolfgang Lukas: Arthur Schnitzler und Tristan Bernard. Anmerkungen zu einem singulären Fall von Doppelgängerschaft. In: Germanica 52 (2013), S. 85-99, sowie dessen Werkartikel in: Christoph Jürgensen/W.L./Michael Scheffel (Hg.): Schnitzler-Handbuch. Leben - Werk - Wirkung. Stuttgart/Weimar: Metzler 2014, S. 96-99. Dort ist auch die weitere Forschungsliteratur verzeichnet. 
Schnitzler spitzt die sich zum Topos auswachsende Doppelzüngigkeit von Journalisten jedoch nicht nur zu, sondern verknüpft sie konsequent mit der vormodernen Logik der Ehre. Damit rückt erneut in den Fokus, dass Verhaltensnormen nicht ohne Reflexion auf den Stand der gesellschaftlichen Entwicklung zu erörtern sind. Auf der Bühne dürfte der soziologische Scharfsinn Schnitzlers seiner wenig gespielten Komödie kaum geholfen haben. Dennoch ist es bemerkenswert, wie Schnitzler mit dem Thema umgeht. Sein Drama macht nicht die Hauptfigur lächerlich, die alternierend „nach jeder Richtung“ schreibt - als Fliederbusch „links“ und als Fink „rechts“ -, sondern diejenigen Kollegen, die sich in Reden und Handeln widersprechen. Ganz nebenbei wird deutlich, dass ein adäquates Verständnis des Relativismus sozialer Rollen nicht zu moralischer Beliebigkeit führen muss. Auch unter den Bedingungen der Moderne sind Komplexität und Unübersichtlichkeit keine Entschuldigung für jegliches Fehlverhalten.

Falsch ist so gesehen nicht, in unterschiedlichen Journalen konträre Meinungen $\mathrm{zu}$ artikulieren. Wenn diese politisch relevant sind und die Austragung zivilgesellschaftlichen Meinungsstreits befördern, sind die beruflichen Rollenerwartungen zweifelsfrei erfüllt, sogar doppelt. Auf diese Weise Geld zu verdienen, ist mithin legitim. Kontextbedingt unterschiedlich zu reden, kann also richtig sein. Tatsächlich falsch ist es dagegen, im selben Kontext anders zu handeln als zu reden. Wer - wie hier - Kritik an überkommenen Ritualen übt, aber den Kollegen mit Perfidie ins Duell treibt, muss sich eine Art Doppelzüngigkeit attestieren lassen und darf kritisiert werden.

Glücklicherweise sind Fink und Fliederbusch eine Person, und die ist keineswegs gesonnen, auf sich selbst zu schießen. So wird das anberaumte Duell zur Szene einer Rekonfiguration. Ähnlich wie bei Freytag dokumentiert Schnitzlers Komödie die dargestellte Entwicklung abschließend durch Neuordnung der Figurenkonstellation. Einerseits lernt die Hauptfigur, die anfangs noch dachte, sich zwischen einer ihrer Identitäten entscheiden zu müssen, mit Unterstützung durch den Grafen Niederhof, dass für journalistische ebenso wie politische Professionalität - „das Technische [eines] Berufs“"18 - Rollendistanz wichtiger ist als Authentizität. Andererseits ordnen sich die Nebenfiguren danach, wie gut es ihnen gelingt, diese Erkenntnis nachzuvollziehen.

Düpiert sind diejenigen, die einen genuin modernen Beruf praktizieren, aber ohne nachzudenken die Verhaltensnormen der ständischen Gesellschaft reproduzieren. Sie reden mit Pathos davon, dass man für seine Überzeugungen einstehen müsse, ohne zu merken, dass ihr Verhalten reproduziert, wogegen sie zu 
kämpfen behaupten. Lachend von dannen ziehen die anderen, die begriffen haben, dass es in den Sphären der Öffentlichkeit, sei es im Parlament, sei es in der Zeitung, darauf ankommt, seine Kräfte „verfügbar zu behalten [...], ohne [...] den größten Teil [der] Seelenkräfte an sentimentale Nebenzwecke verschwenden zu müssen “. ${ }^{19}$ Andernfalls finden berechtigte Meinungen kein ausreichendes Gehör und objektive Zwecke werden durch subjektive Bedürfnisse verdrängt. Auch Authentizität, die in anderen Zusammenhängen durchaus geboten sein kann, ist hier allenfalls der Name einer Partei. Wer das begriffen hat, kann sich weiter Redeschlachten liefern. Aber wenn man die dafür vorgesehenen Foren verlässt, dann geht man im besten Fall zusammen essen. Duellieren wird man sich sicher nicht. ${ }^{20}$

\section{Zwischenbilanz mit Seitenblick auf Hans Natonek: Kinder einer Stadt}

Zieht man eine Zwischenbilanz, dann fällt auf, dass bislang die Subjekt-Seite der journalistischen Tätigkeit im Vordergrund stand. Der Zugang zum Thema erfolgt über die Person des Journalisten und seine Tätigkeit, die Meinungsproduktion. Dabei ist die Tätigkeit an die Person zurückgebunden. Texte und Figuren stellen die Hintergrundmotivation zur journalistischen Arbeit und das konkrete Vorgehen der jeweiligen Journalisten auf den Prüfstand.

Selbst Schnitzler stellt die Journalisten-Figuren als fiktive Personen dar, deren Handeln auf der Theaterbühne untersucht und bewertet wird. Am Ende kehrt sich bei ihm die Perspektive jedoch um. Journalisten werden nun nicht mehr über einen Kamm geschoren, sondern teilen sich in zwei Gruppen: solche, die bewertet werden, und solche, die den Maßstab dafür abgeben. Exemplarisch für alle Berufe, zu deren Anforderungsprofil es gehört, eine öffentliche Rolle zu spielen, erscheint der richtig verstandene Journalist als Allegorie moderner Un-

19 Schnitzler: Fink und Fliederbusch (Anm. 15), S. 202.

20 Vgl. Schnitzler: Fink und Fliederbusch (Anm. 15), S. 203: „GRAF beiläufig Überzeugung -! [...] Überzeugung -?! Wo gibt es überhaupt im Einzelfall einen strikten Beweis dafür, daß eine wirkliche Überzeugung vorhanden ist - und nicht eines ihrer zahlreichen täuschend ähnlichen Surrogate. / Fliederbusch fein Es gibt einen Beweis, Herr Graf. Bereit zu sein - für diese Überzeugung - zu sterben. GRAF lächelnd Ach so. - Aber das ist doch manchmal - Sie verzeihen schon, Herr Fink -, manchmal sag ich - auch nur eine Art, den anderen - oder sich selber weiszumachen, daß man eine Überzeugung gehabt hat.“ 
eigentlichkeit und Kriterium, an dem sich zeigt, inwieweit Akteure die Bedingungen ihres Handelns verstehen.

Dank dieser Verkehrung kann man Schnitzlers Drama entnehmen, worin das Unbehagen gegenüber dem Journalismus wurzelt. Die Exposition des Journalisten als Figur der Uneigentlichkeit impliziert, was den Generalverdacht gegen ihn motiviert. Es ist einmal mehr das Unbehagen an einer Moderne, die alle Unmittelbarkeit auszehrt. ${ }^{21}$ Abstraktheit, Komplexität sowie Uneigentlichkeit, Unübersichtlichkeit und andere Verlustanzeigen bezeichnen verschiedene Facetten einer kulturellen Grundstruktur. Wer auf Journalisten zielt - und nicht einfach ein Alleinherrscher ist, der Andersdenkende mundtot macht -, der verleugnet die Unhintergehbarkeit dieser kulturellen Grundstruktur in strategischer Absicht. Aus dem weit verbreiteten Unbehagen an der hochgradigen Vermitteltheit der Welt versucht das Journalisten-Bashing Kapital zu schlagen, indem es einen Berufsstand dafür verantwortlich macht, dass niemand mehr ,klar' sieht.

Was allem Leidensdruck zum Trotz nicht auf Handlungen und Entscheidungen zurückgeführt werden kann, dass nämlich die Welt sich unmittelbarem Zugriff entzieht und nur als ein ,Welt-Bild“ unter vielen zu haben ist, von denen jedes unverkennbare Spuren einer Herstellung an sich trägt, soll denjenigen in die Schuhe geschoben werden, die aus der Not der Mittelbarkeit eine Tugend gemacht haben, den sogenannten ,Medien'. Eine Personengruppe aber, die stellvertretend für die Bedingungen, denen sie ihre professionelle Existenz allererst verdankt, verantwortlich gemacht wird, kann kaum anders als durch und durch negativ charakterisiert werden. So lässt sich erklären, wieso die persönliche Unzuverlässigkeit das topische Stigma beinahe jedes literarisch dargestellten Journalisten ist, während das Moment der Herstellung beziehungsweise Konstruktion die Abneigung gegen die professionelle Meinungsproduktion entfesselt.

Schnitzler beweist zwar, dass man Uneigentlichkeit als eine moderne Rollenerwartung, mit der nicht nur Journalisten und Politiker konfrontiert sind, begreifen, bejahen und sogar differenziert von Unaufrichtigkeit abheben kann, aber es verlangt nur wenig Fantasie, sich vorzustellen, wie wenig Resonanz er damit gefunden hat. Zu verführerisch ist es, dass der Journalist als Agent der Moderne, deren Komplexität so schwierig zu durchdringen ist, genau die Eigenschaften verstärkt, die es so schwer machen, die Moderne zu mögen. Dieses Verhältnis zur Moderne, die Repräsentation ihres verhassten Teils, disponiert den Journalisten

21 Vgl. auch das Kapitel „Soziologische Trauerarbeit“ in Ingo Stöckmann: Der Wille zum Willen. Der Naturalismus und die Gründung der literarischen Moderne 1880-1900. Berlin/New York: De Gruyter 2009, S. 270ff. 
zum Sündenbock. Daher ist es kein Zufall, dass er häufig als Jude dargestellt und in ein antisemitisches Licht getaucht wird.22

In der Literatur der Weimarer Republik spitzt sich diese Konstellation erwartungsgemäß zu. Gut zu sehen ist das an dem in Vergessenheit geratenen, aber gelegentlich daraus auftauchenden Roman Kinder einer Stadt (1932) von Hans Natonek..$^{23}$ In dessen zweitem Teil steht im Zentrum ein jüdischer Journalist, der sich in den Nachkriegswirren eine neue Identität erschafft, seinen Körper stählt, von Prag nach Hamburg übersiedelt, ins antisemitische Lager wechselt und seine ehemaligen Schulkameraden, allesamt Journalisten, mit unlauteren Mitteln bekämpft. An dieser Figur des Jakob Dowidal, später Arnold Widahl, zeigt sich wie in einem Brennglas das Knäuel der verschiedenen roten Fäden des Problems. Seine Lebensgeschichte ist genuin modern, weniger in ihrem Leiden an sozialer Ausgrenzung als in den Künsten ihrer Überwindung. Diametral entgegensteht der performativen Modernität der programmatische Anti-Modernismus und Antisemitismus, der die liberalen Prinzipien verächtlich macht, auf denen die Karriere des Sprechers gründet.

Doch damit nicht genug: Sein biografischer ,Konstruktivismus‘ bleibt anfällig für authentische Informationen über die ,wahre Herkunft' gerade dann, wenn die Kultur der Moderne abgelehnt und der Wert rassischer und genealogischer Information fortwährend beteuert wird. Daher muss Dowidal/Widahl sich Sorgen machen, als zwei seiner ungleichen Jugendgefährten nach dem Weltkrieg und trotz Ortswechsel zufällig wieder in seine Nähe kommen. Vor allem aber bietet sich die Chance, ein nicht ausgetragenes Duell mit anderen Waffen nachzuholen. Der Angriff von Dowidal/Widahl zielt zwar auf den Ruf seines Kon-

22 Signifikant für die Logik der Verdächtigung ist auch, dass diejenigen, die bekanntermaßen wissen, dass es keine privilegierte Perspektive auf die Welt gibt und in Zusammenhang damit oft als Überläufer figurieren, an anderer Stelle bezichtigt werden, uns ihre Perspektive zu oktroyieren.

23 Seit Kurzem bemüht sich der Lehmstedt-Verlag in Leipzig um die Wiederentdeckung der Publizistik des Feuilleton-Journalisten der Weimarer Republik und späteren Emigranten Hans Natonek. Vgl. Hans Natonek: Im Geräusch der Zeit. Gesammelte Publizistik 1914-1933. Hg. von Steffi Böttger. Leipzig: Lehmstedt 2013; Hans Natonek: Letzter Tag in Europa. Gesammelte Publizistik 1933-1963. Hg. von Steffi Böttger. Leipzig: Lehmstedt 2013. Steffi Böttger hat auch eine Biografie zum Autor vorgelegt. Zum Autor siehe auch Jürgen Serke: „Hans Natonek“. In: J.S.: Böhmische Dörfer. Wanderungen durch eine verlassene literarische Landschaft. Wien/Hamburg: Zsolnay 1987, S. 86-129. Aus der spärlichen Forschungsliteratur vgl. das allerdings primär auf den ersten, ,Prager‘ Teil abzielende Kapitel von Kristina Lahl: Das Individuum im transkulturellen Raum. Identitätsentwürfe in der deutschsprachigen Literatur Böhmens und Mährens 1918-1938. Bielefeld: transcript 2014, S. 262-283. 
trahenten Epp, aber er vollzieht sich nicht im Regelkreis der Ehre, ${ }^{24}$ sondern bedient sich einer Pressekampagne - also abermals genau der Mittel, die die von ihm bekämpfte Kultur zur Verfügung stellt. Und nicht nur das. Er wartet nicht erst auf Fakten, sondern schafft sie selbst. Zu guter Letzt muss Epp in die gestellte Falle noch nicht einmal vollständig tappen, um von der anschließenden Medienkampagne zermalmt zu werden. Dowidal/Widahl konstruiert aus Epps persönlichem Makel, jeder Frau nachstellen zu müssen, ein Versagen des liberalen Systems und stülpt die Diagnose wiederum dem Einzelnen über den Kopf, bis es ihm die Luft zum Atmen nimmt.

Als der mit einer Minderjährigen leicht zu ködernde Epp von Dowidal/Widahl zugrunde gerichtet ist, nimmt er das Wissen von der Herkunft seines Gegners mit ins Grab. ${ }^{25}$ Weil Epp fair - um nicht zu sagen ehrenhaft - kämpfen wollte und als Vertreter eines modernen liberalen Weltbildes keinen prinzipiellen Vorbehalt gegen eine Selbsterfindung im Stile von Dowidal/Widahl hegt, hat er sein geheimes, aber authentisches Wissen über den anderen nicht in Anschlag gebracht. ${ }^{26}$ Doch der Preis für diese Rücksichtnahme ist hoch. Am Ende unterliegt er einer Intrige, bei der die wichtigste Anschuldigung der Pressekampagne gegen ihn von einem Journalisten absichtlich erzeugt wurde, der sich politisch als strammer Kämpfer gegen die Laster der Moderne profiliert.

Die Komplementarität der Kontrahenten ist offensichtlich. Doch die semantische Ausstattung ihrer Gegnerschaft durch den Text ist alles andere als eine paradigmatisch reine Opposition. Vielmehr verschränken sich auf beiden Seiten Eigenschaften der kulturellen Moderne mit Merkmalen vormoderner Interaktionskulturen. Authentische Informationen, deren Veröffentlichung in der Presse von Gewicht wäre, werden zurückgehalten, während von programmatisch anti-moderner Seite aus alle Register des genuin modernen Kampfes um die öffentliche Meinung gezogen werden, bis hin zur Fälschung.

Mit seinem Katalog journalistischer Stile und Motivationen, der noch um zwei Personen erweitert werden müsste, eignet sich Natoneks Roman Kinder einer Stadt gut für einen Abschluss des historisch ersten Paradigmas der literarischen Journalismusdarstellung. Instruktiv ist ein kurzer Vergleich zwischen Schnitzlers

24 Vgl. zur literarischen Diagnose der Auflösung des Ehrparadigmas um 1900 Matthias Schöning: Anerkennung und Ehre. Literarische Mikrosoziologie und Ehr-Kritik um 1900: Schnitzler, Sudermann, Fontane. In: Martin Baisch (Hg.): Anerkennung und die Möglichkeiten der Gabe. Literaturwissenschaftliche Beiträge. Frankfurt/M. u.a.: Peter Lang 2017, S. 121-154. 25 Von einer weiteren Person, Waisl, ebenfalls Jugendgefährte und Angestellter Dowidals beziehungsweise Widahls, muss hier abgesehen werden.

26 Vgl. Hans Natonek: Kinder einer Stadt. Wien/Hamburg: Zsolnay 1987, S. $254 f f$. 
Drama Fink und Fliederbusch auf der einen Seite und Hans Natoneks Roman Kinder einer Stadt.

Wie Schnitzlers Handlungsführung mit ihrer Neukonfiguration der Figurenkonstellation am Schluss demonstriert, hängt gute journalistische Arbeit nicht davon ab, dass Personen authentisch verkörpern, was sie in ihrer beruflichen Rolle kommunizieren. Das Kriterium guter journalistischer Praxis ist vielmehr eine Kommunikation, die insofern intellektuell wahrhaftig ist, als sie ihrer medialen und gesellschaftlichen Strukturvoraussetzungen angemessen ist. Selbstironisch bleibt freilich der Schluss. Er sollte nicht so gelesen werden, als könne man mit dem abstrakten Kriterium alle konkreten Protagonisten eindeutig schwarz oder weiß zuordnen.

Hier setzt Natoneks Roman an, der Schnitzlers Differenzierung seitens Text und Leser gleichsam voraussetzt, um an den Figuren eine Abstufung von Grautönen darzustellen. Der Erfolg von Dowidal/Widahl zum Beispiel resultiert daraus, dass er versteht, wie man unter Bedingungen moderner Öffentlichkeit eine Kampagne startet. Er lässt politisches Programm und persönliches Verhalten seines Kontrahenten in einer Art Mythos verschmelzen, in dem zwischen liberalen Prinzipien und prinzipienloser Libertinage kein Unterschied mehr besteht. Unredlich an seiner Kampagne ist allerdings nicht so sehr die kalkulierte Entdifferenzierung zwischen privatem Verhalten und öffentlicher Rolle seines Gegners Epp. Die medienethische Frage, die dadurch aufgeworfen wird, dass Epp sein privates Wissen ausdrücklich nicht nutzt, veranschaulicht das Spektrum journalistischer Stile und stellt die den einzelnen Journalisten betreffende Frage, welches Berufsethos man pflegen will.

Inkriminiert wird die Kampagne von Dowidal beziehungsweise Widahl erst dadurch, dass sie den Gegenstand der Berichterstattung intentional erzeugt. Während Epp Tatsachenwissen nicht nutzt, weil er die Grenzen zwischen Privatsphäre und Öffentlichkeit respektiert, lässt Dowidal/Widahl nicht nur genau diese Grenze kollabieren, sondern er erfindet sich den Anlass seiner Kampagne. Erst damit wird die Figur final als unredlich markiert und der schwerwiegende, für die Praxis des Journalismus insgesamt fundamentale Verdacht profiliert, dass die vermeintlichen Fakten bei Bedarf eigens hergestellt werden. Jetzt ist das Maximum dessen erreicht, was im ersten Paradigma der Journalismus-Darstellung als Vorbehalt formuliert werden kann. 


\section{Heinrich Böll: Die verlorene Ehre der Katharina Blum}

Eine grundsätzlich veränderte Problemlage, die ein zweites darauf aufbauendes Paradigma kennzeichnet, findet man erst erheblich später. Wie sich die Problematik über Fragen der Figurencharakteristik und der journalistischen Praktiken hinaus ausweitet, kann man prominent an Heinrich Bölls Novelle Die verlorene Ehre der Katharina Blum aus dem Jahre 1974 studieren. Auch Böll stattet seine Journalistenfigur(en) mit erheblicher Unredlichkeit aus, die Pointe seines Textes liegt jedoch woanders. Was ihm in der Realität einer vom Terrorismus heimgesuchten Bundesrepublik nicht gelingt, funktioniert in der fiktiven Welt seiner Erzählung sehr gut: Man sieht, wie sich der Journalismus die Welt, aus der er vermeintlich nur berichtet, in gewissem Maße selbst erschafft - und zwar unbemerkt, nicht-intentional. Damit erklimmt die Problemlage ein neues Niveau.

Im Gleichschritt mit der fortschreitenden Modernisierung wird aus dem stellvertretenden Sündenbock für die Schattenseiten der Moderne einer der Hauptverursacher ihres sich ausweitenden Übels. Hieß es zunächst, dass die in den Händen unzuverlässiger Personen befindliche und insofern willkürliche $M e i$ nungsproduktion den Blick auf die eigentliche Welt verstellt - im schlimmsten Fall durch bewusste Desinformation -, so lautet die Anklage nun auf Installation eines undurchschauten Systems der nicht-intentionalen Wirklichkeitserzeugung. Dieser Vorwurf ist insofern von größerem Gewicht, als er den Journalismus nicht nur bezichtigt, eine Agentur der ,Ent-Wirklichung` zu betreiben, sondern letztlich eine Herrschaft der Medien postuliert, die das Reale längst verdrängt hat und alles, sich selbst eingeschlossen, in einer Selbstreproduktionsschleife gefangen hält.

Was nach Jean Baudrillard klingt, findet sich als Vorstufe tatsächlich auch bei Böll. Dieser hatte wiederholt versucht, die Springer-Presse in Mithaftung zu nehmen für die Atmosphäre der Gewalt, die der Linksterrorismus der Siebzigerjahre verbreitet. Sein Spiegel-Essay „Will Ulrike Gnade oder freies Geleit?“27 insinuiert daher einen kausalen Einfluss der Berichterstattung auf das Terroristenhandeln. Dagegen sind mit gutem Recht Einwände erhoben worden. ${ }^{28}$ Man kann zwar eine kalkulierte Hysterisierung der Öffentlichkeit durch die Berichterstat-

27 Vgl. Heinrich Böll: Will Ulrike Gnade oder freies Geleit? In: Der Spiegel, Nr. 3 von 10. Januar 1972, S. 54-57.

28 Vgl. Sonja Krebs: Rechtsstaat und Pressefreiheit in Heinrich Bölls Die verlorene Ehre der Katharina Blum. Ein Beitrag zu Verfassungstheorie und Verfassungswirklichkeit im Spiegel der Literatur. Diss., Mainz 1990, S. 22-35. 
tung der BILD-Zeitung diagnostizieren, einen kausalen Wirkungszusammenhang $\mathrm{zu}$ behaupten führt jedoch deshalb zu weit, weil er die Verantwortlichkeit für konkrete Straftaten verschiebt. Im fiktiven Szenario der Novelle funktioniert dieselbe Hypothese eines verdichteten Wirkungszusammenhangs von Journalismus und Mord jedoch sehr gut und entfaltet eine durchaus erhellende Wirkung.

Nachdem der Beginn des Textes deutlich gemacht hat, dass er sich explizit zu journalistischer Arbeit ins Verhältnis setzt, ${ }^{29}$ läuft die dargestellte Handlung darauf hinaus, den Mord der Katharina Blum am Boulevard-Journalisten Werner Tötges - nomen est omen - als Folge von dessen journalistischen Nachstellungen erscheinen zu lassen. Der vollständige Titel signalisiert diese Absicht: Die verlorene Ehre der Katharina Blum oder: Wie Gewalt entstehen und wohin sie führen kann. ${ }^{30}$ Dabei muss jedoch festgehalten werden, dass Katharina Blum nicht bloß auf eskalative Weise Rache für die unfaire Berichterstattung über ihre Person und die ihr zu Last gelegte Fluchthilfe übt. Mit einer solchen, bloß die Affektlage einer Figur vorführenden Darstellung begnügt sich der Text nicht. Vielmehr stellt er sowohl Blum als auch Tötges als Rädchen eines von beiden nicht durchschauten Wirkungszusammenhangs dar, infolgedessen der Boulevard-Journalismus die Gewalt, von der seine Aufmacher vermeintlich nur zeugen, selbst reproduziert. Die Gewalt, die von der Presse ausgeht, kehrt sich nicht lediglich um und richtet sich nun gegen einen ihrer Repräsentanten. Wichtiger ist, dass das Wuchern der Gewalt die Selbst-Reproduktion der Presse auf gleichbleibend drastischem Aufmacherniveau garantiert. ${ }^{31}$

Dabei spielt insbesondere die als Leitmotiv eingesetzte Wohnung eine wichtige Rolle - und damit abermals die Unterscheidung von privatem und öffentlichem Raum. Vormals sicherer Rückzugsraum einer jungen, aber bereits geschiedenen, um Selbständigkeit besorgten Frau, wird plötzlich alle Privatsphäre durch die Berichterstattung über sie zerstört. Nachdem die Pressekampagne begonnen hat, wird ihr von verhetzten Lesern derart nachgestellt, dass sie in den eigenen vier Wänden, für deren Anschaffung sie so viel gearbeitet hat, nicht mehr sicher ist. Mit der Zerstörung ihres Refugiums wird ihr regelrecht die Luft zum Atmen

29 Der erste Satz lautet: „Für den folgenden Bericht gibt es einige Neben- und drei Hauptquellen, die hier am Anfang einmal genannt, dann aber nicht mehr erwähnt werden." Heinrich Böll: Die verlorene Ehre der Katharina Blum oder: Wie Gewalt entstehen und wohin sie führen kann. München: DTV 1985, S. 7.

30 Zu Titel und Prätexten vgl. Klaus Jeziorkowski: „Die verlorene Ehre der Katharina Blum oder: Wie Gewalt entstehen und wohin sie führen kann“. In: Werner Bellmann (Hg.): Heinrich Böll, Romane und Erzählungen. Stuttgart: Reclam 2000, S. 249-268.

31 Böll: Katharina Blum (Anm. 29), S. 12: „Ziemlich merkwürdig verhielt sich die ZEITUNG, nachdem die beiden Morde an ihren Journalisten bekannt wurden. Irrsinnige Aufregung! Schlagzeilen. Titelblätter. Sonderausgaben. Todesanzeigen überdimensionalen Ausmaßes.“ 
genommen. Indem sie den Journalisten, der dafür verantwortlich ist, in eben diese Wohnung bestellt und dort erschießt, passt sie sich nicht so sehr an das Bild an, das die Zeitung von ihr gezeichnet hat. Vielmehr setzt sie in der bereits entweihten Wohnung einen Schlusspunkt unter ihr in die Öffentlichkeit gezerrtes Leben und zieht sich symbolisch an den einzigen Ort zurück, der noch etwas für sie Wertvolles beherbergt - das Gefängnis, in dem auch der inzwischen gefasste und von ihr geliebte Straftäter einsitzt.

Indem Böll einen solchen Wirkungszusammenhang skizziert, bietet er ein frühes Beispiel für eine fundamentale Verschiebung des Vorbehalts gegenüber Journalisten und Journalismus - wenngleich hier noch in einer begrenzten Form, die vor allem auf den Boulevard-Journalismus abzielt. ,Klassisch' ist der Verdacht, dass Journalisten unredlich arbeiten und die Fakten entstellen. Neu ist der Vorbehalt, dass sie die Welt, aus der sie vermeintlich nur berichten, nicht-intentional (mit-)erschaffen. Das Gewicht liegt auf der Nicht-Intentionalität. Weil sie nicht durchschauen, dass sie sich ihre eigene Welt erschaffen, sind sie - und die Leser mit ihnen - in ihr gefangen. Das unterscheidet das Szenario bei Böll von dem Natoneks. Während dort ein identifizierbarer Gegner mit persönlicher Motivation seinem Feind eine spezifische Falle stellt und für die anschließende Kampagne die benötigten ,Fakten` schafft, kommt bei Böll zu dieser ersten Stufe noch eine zweite hinzu, auf der sich die Erzeugung der immer neuen Schlagzeilen von Personen und personalen Motivlagen ablöst. Tötges hat natürlich zu keinem Zeitpunkt intendiert, selber getötet zu werden, um seinen Kollegen neues Material zu liefern. ${ }^{32}$

Heinrich Böll, von dem man das vielleicht nicht vermutet hätte, liefert mit seiner Novelle Die verlorene Ehre der Katharina Blum eine Beschreibung auf der Höhe seiner Zeit. Im Vorgriff auf konstruktivistische Medientheorien skizziert er die Gefahr einer in den eigenen Konstruktionen eingeschlossenen Medienmaschine. Bölls Grenze besteht allerdings darin, dass er dem Kritiker einen Standort außerhalb reserviert. Wie man an seinem Ulrike Meinhof-Essay sieht, geht er von der Annahme eines fixen Verantwortungsquantums aus, das zwischen verschiedenen Akteuren, vor allem Terroristen, Presse, Polizei und Justiz zu verteilen ist. Damit hält er an einer gleichsam monopolaren Welt fest, in der sich definitiv bestimmen lässt, wer wieviel Verantwortung für etwas trägt.

32 Dass diese den Mord am Kollegen gleichwohl ausschlachten, versteht sich von selbst. Bölls Text nennt es die „Überaufmerksamkeit der Presse“, die vermerkt werden müsse. Böll: Katharina Blum (Anm. 29), S. 12. 


\section{Josef Haslinger: Opernball - und Schluss}

Auf den Spuren der Postmoderne geht Josef Haslinger in seinem Roman Opernball den letzten entscheidenden Schritt weiter. ${ }^{33}$ Haslingers Text ist nach Art einer Tiefenstruktur eine nahezu systematische Stufenleiter journalistischen ,Worldmakings' hinterlegt, die mit der Differenz zwischen der Berichterstattung von staatlichen und privaten Medienunternehmen beginnt, verschiedene Modi der Kriegsberichterstattung einschließt und mit nicht-intentionalen Medieneffekten endet. Weil der Erzähler hier ein Fernsehjournalist ist, ist der qualitative Sprung von intendierten Fälschungen des Bildmaterials - zum Beispiel eine Umkleidung, die aus toten Soldaten tote Zivilisten macht - zu nicht-intendierten Medieneffekten einfacher zu fassen als bei Böll. Zivilisten, Polizisten und Terroristen verhalten sich gleichermaßen anders, sobald Kameras ins Spiel kommen. Die Zivilisten fühlen sich in der Konfrontation mit der Staatsmacht stärker geschützt und werden provokativer. Die Polizisten nehmen sich mit Blick auf ihr Bild in der Öffentlichkeit stärker zurück. Und für die Terroristen ist die Anwesenheit von Kameras eine notwendige Bedingung dafür, überhaupt an dieser Stelle loszuschlagen. „Die Mechanismen eines den eigenen Aktualitätsreflexen folgenden Live-Fernsehens" haben das Handeln aller infiziert. ${ }^{34}$

Diese Medieneffekte sind ursprünglich von niemandem intendiert. Man berichtet nicht, um diese Nebenwirkungen zu erzielen. Doch wenn man sie erst einmal kennt, sind Versuche der Instrumentalisierung unausweichlich. Allerdings führen sie aus der Reihe der Medieneffekte nicht hinaus, sondern verlängern sie nur. Im Zeitalter der Handyfilme, von denen Haslinger noch nichts wissen kann, potenziert sich die Kette der Effekte ins Unendliche. Die mediale Ausleuchtung ist potenziell panoptisch. In der Welt des Romans braucht es noch Übertragungswagen und tausende Meter Kabel für die Liveberichterstattung vom Opernball, der zum Ziel eines Terroranschlages mit Giftgas werden wird. Doch schon hier werden nicht-intendierte Medieneffekte bereits ins Kalkül einbezogen, und der Roman weiß auch schon, dass sie dadurch nicht durchbrochen, sondern nur komplexer werden.

Der Text trägt dem dadurch Rechnung, dass er das unübersichtliche Geflecht wechselseitiger Bedingungen klar evoziert, aber zu keinem einzelnen

$33 \mathrm{Vgl}$. dazu - mit weiteren Literaturhinweisen - Rüdiger Wischenbart: [Eintrag] Haslinger, Josef. In: Munzinger Online/KLG - Kritisches Lexikon zur deutschsprachigen Gegenwartsliteratur: http://www.munzinger.de/document/16000000210 (zuletzt eingesehen am 21.03.2017).

34 Harro Segeberg: 'Retour à la Réalité'? Nebel, Baudrillard und die Metaphysik antimedialer Revolten. In: François Poncet (Hg.): Gerhard Nebel. „Ein gewaltiger Verhöhner des Zeitgeistes“. München: Fink 2013, S. 135-150, hier S. 148. 
Bedingungspol hin auflöst. Dem entspricht eine Handlung, die bis zum Ende ungeklärt lässt, wie weit die beunruhigende Komplizenschaft zwischen Medien und Attentätern geht. Schwache Indizien erzeugen explizit den Verdacht, dass ganz oben in der Hierarchie des übertragenden Fernsehsenders jemand Informationen über das Frischluftsystem der Wiener Staatsoper weitergegeben haben könnte. Gänzlich unklar bleiben die Intentionen; wenn es denn überhaupt so gewesen sein sollte. Vielleicht haben die Terroristen den Opernball aber auch nur deshalb ausgewählt, weil die durch den Privatsender gegenüber den Vorjahren erhöhte Übertragungsintensität eine erhöhte Aufmerksamkeit auch für den Terror nach sich zieht. Ihr Kalkül wäre dann jedenfalls aufgegangen: Die Live-Berichterstattung vom Massensterben läuft selbst nach dem Tod der Kameraleute weiter. Diese Komplizenschaft der Medien ist in jedem Fall unvermeidlich.

Der Coup des Romans resultiert aus dem genauestens abgewogenen Spiel mit gemutmaßten Wechselwirkungen zwischen den verschiedenen Akteuren, die intradiegetisch als nachgeordnete Erzählerstimmen präsent sind. Einer leichten Inkonsequenz könnte man die Rahmenkonstruktion bezichtigen. Deren Erzähler, ein Fernseh-Journalist, der nach verschiedenen Stationen als Kriegsberichterstatter erstmalig die Übertragung vom Opernball leitet, die sich der Privatsender, zu dem er im Laufe seiner Karriere gewechselt ist, mit viel Geld gesichert hat, verliert bei der Katastrophe seinen Sohn. Seine Trauerarbeit ist die investigative Recherche, die nun nicht aus Bildern, sondern aus auf Tonband aufgezeichneten Berichten besteht. Deren Einheit wiederum bildet, integriert durch seine eigene Icherzählung, der Roman Haslingers. Zwar führt die Investigation zu keinem eindeutigen Ergebnis, sondern dem Bild eines unauflöslichen Bedingungsgeflechts. Die Rückkehr zum investigativen Journalismus, zumal einem bildlosen, scheint jedoch einen Rest an Hoffnung zu implizieren, es könne eine Wahrheit jenseits der medial erzeugten Welten geben.

Für einen anti-kritischen Impuls gegen die generalisierende JournalismusKritik ist das jedoch ein Vorteil. Anstatt die Beweisaufnahme für eine totale Gefangenschaft in den Selbstreproduktionsschleifen einer entfesselten Medienwelt für abgeschlossen zu erklären, stellt sich die Frage neu, mit welchem Konzept an die zur Medien-Frage erweiterte Journalismus-Frage heranzugehen ist: Was ist besser? Dürfen wir davon ausgehen, dass es auch in einer Welt, die dominant medial konstruiert ist, immer eine Lücke gibt, durch die wir - vielleicht unter Schmerzen - mit einer unkonstruierten Welt in Kontakt kommen können? Einzelne Fragen können dann abschließend geklärt werden, sicher nicht immer, aber prinzipiell. Oder müssen wir annehmen, dass sich Konstruktionen von Wirklichkeit immer nur gegen andere Konstruktionen austauschen lassen? Auch dann ist ,Wahrheit nicht obsolet, aber die Wahrheitstheorien sind komplexer und 
krisenanfälliger. Und vielleicht lassen sie sich auch temporär entwenden, um damit ,postfaktische' Politik zu betreiben.

Am Ende dieses Parforceritts kann man immerhin sagen, dass die Frage nach der Totalität der Medialisierung, die von der Postmoderne bereits für beantwortet erklärt worden war, wieder aufgenommen werden sollte. Wie man sich erkenntnistheoretisch positionieren will, war hier nicht die Frage. Sicher ist folgendes: Wir leben in hochgradig konstruierten Welten. Der Journalismus und alle anderen ,Medien' sind ein starker Faktor unserer Weltkonstruktion. Und sie wären es auch dann, wenn es bei ihnen stets mit rechten Dingen zuginge, was sicher niemand behaupten will. Intentionale und nicht-intentionale Verzerrungen im Zuge der medialen Wirklichkeitskonstruktion rechtfertigen jedoch nicht, den Journalismus oder die Medien per se zu verdammen oder journalistische Arbeit und schließlich sogar Journalisten als Personen zu verunglimpfen und anzugreifen. Wer glaubt, der Journalismus und andere gesellschaftliche Institutionen der Weltkonstruktion würden den Blick auf die eigentliche Welt zustellen, anstatt Perspektiven zu eröffnen, laboriert an einem anderen, grundlegenderen Problem. Er bedauert, dass es mehr als Perspektiven nicht geben kann, egal wie laut man das Gegenteil behauptet. Der Zugang zur Welt, selbst wenn es ein Zugang zur Welt selbst sein sollte, ist insofern immer vermittelt - Unmittelbarkeit aber ein Mythos der Moderne.

Die Journalismus-Kritik ist nicht nur eng mit der Geschichte des Antimodernismus verknüpft. Deren Muster weisen eine Parallelität auf, die eine wichtige Schlussfolgerung für kritische Beobachter enthält: So wie der klassische Antimodernismus die Kultur der Moderne und deren Selbstreflexion als Existenzbedingung und intellektuell voraussetzt, so setzt auch die radikalisierte Kritik der Medien mit Schlagworten wie ,Lügenpresse die postmoderne Kultur und deren Reflexionsgewohnheiten voraus. So gesehen ist es weniger vordringlich, selbstkritisch mit der Postmoderne abzurechnen, als vielmehr an der Zeit, die Theoriebildung dialektisch voranzutreiben. 